\title{
A Real-time Automatic Instrument Tracking System on Cataract Surgery Videos for Dexterity Assessment
}

\author{
Vassilis Baldas, Member, IEEE, Lilian Tang, Panagiotis Bountris, \\ George Saleh and Dimitrios Koutsouris, Senior Member, IEEE
}

\begin{abstract}
In this paper we describe the SUITS (Surrey University Instrument Tracking System), an automated video processing system that analyzes videos of cataract surgeries to extract parameters for surgical skill assessment. Through image processing and object tracking techniques the eye is identified, and its movement and direction changes throughout the operation are monitored. Any instrument that moves into or out of the eye is located with its path measured. So far we have developed a prototype real-time system that has demonstrated great potential. The developed system is automatic, with minimal human supervision required throughout the processing time. In addition, the solution is generic, and it can be applied to other tracking problems, possibly other types of surgery videos, with minor modifications.
\end{abstract}

Index Terms - Instrument tracking, cataract videos, segmentation, real-time, surgeon dexterity assessment

\section{INTRODUCTION}

$\mathrm{T}$ HE style of medical training has emphasized more on standardized and objective assessment of clinical, academic and surgical knowledge. Traditionally in ophthalmology, surgical skills are often assessed in the operating theatre environment with the supervising surgeon directly observing or providing feedback whilst watching a recording of the operation. This can be of great subjective variability and is not readily reproducible. Certain components of surgical skills can be determined by analyzing the movement of the instruments. For example, the total number of movements, the path length, the time taken for the task and other motion parameters are found to correlate well with the experience of surgeon. A technically good surgeon is one perceived as being both quick and displaying greater economy and precision of movement [1].

Attempt has been made on motion tracking using the Imperial College Surgical Assessment Device (ICSAD), which allows meticulous interrogation of performance, including a detailed analysis of movement behaviour [2]. Tracking is

Manuscript received July 15, 2010

Vassilis Baldas is with the Biomedical Engineering Laboratory, National Technical University of Athens, Greece. tel . +302107722286 baldas@biomed.ntua.gr

Lillian Tang is with the Department of Computing, University of Surrey, UK. H. Tang@surrey.ac.uk

Panagiotis Bountris is with the Biomedical Engineering Laboratory, National Technical University of Athens, Greece. pbountris@biomed.ntua.gr

George Saleh is with Moorfields Eye Hospital, London, UK. George.Saleh@moorfields.nhs.uk

Dimitrios Koutsouris is with the Biomedical Engineering Laboratory, National Technical University of Athens, Greece. dkoutsouabiomed.ntua.gr performed through placing probes on the hand and tracking the movement. That system, however, did not take into consideration what is going on at the core of the operation. What surgeons care most about is not the hands but actually what the results of the hands action are i.e. the instrument movements. It would be a more direct measure if the recorded videos can be automatically assessed through tracking and analyzing the movement of surgical instruments. Apart from evaluating surgical performance an automated instrument tracking system potentially can offer real-time feedback to the surgeon during the operation. It is towards these possibilities and concerns that this research is directed.

\section{OBJECTIVES OF THIS WORK}

The main objective of this work named SUITS (Surrey University Instrument Tracking System) is to develop an automated computational system that analyzes videos of cataract surgeries in order to track the position of the surgical instrument in every part of the surgery. The knowledge of the path that the instrument follows can be used afterwards to extract higher level information.

For intraocular surgery, there are hundreds of different instruments. However there is only a maximum of two in the eye at any given time. The operation is divided into several stages. For each step there is a wide variety of specific instruments (i.e. many different shapes, sizes, designs all for a specific task e.g. - numerous injectors to insert the lens - numerous different instruments are used to manipulate the lens etc. In terms of their major impacts on the visual content in video recordings, commonly used object tracking methods are not immediately applicable due to the following issues which need to be addressed by the system:

- The insertion and withdrawal of any instrument. This requires the identification of new object and adaptive ability to trace the objects with various speeds when it is appearing to and disappearing from the scene. During the operation, the instrument movement can be sometimes slow and sometimes fast when performing key operations such as cutting, removing, sucking, holding etc. In many occasions the movements can be very subtle, thus require a precise measurement of the tip.

- Large number of different instruments. Since each surgeon can have his own set of instruments, the characteristics of instruments to be tracked cannot be predefined. A general tracking solution has to be proposed to deal with this variation. 
- The Movement of the eye. If tracking is targeted at the surgical instruments, the eye will be typically treated as a background, which is constantly moving. This adds extra complexity to the solution of the problem. On operating different patients, the various size, colour, shape of the eye, clinical conditions will give rise to a very diverse background.

- Occlusion of Instruments and the Eye. Some occlusions are also transparent. For example, before and after inserting the instrument to different layers of the eye, e.g., the capsule, and interacting with the eye, the intensity information of the instrument can change due to such transparent occlusion.

- Varied Video Quality. Different clinical units use different video recording equipments and the quality varies across collected videos. In those poor quality videos often a great deal of noise are present.

- Moving Camera: . In most of the videos, the camera moves along with the surgeons hand.

- Other Variations. There are changes in magnification, size in different videos, illuminations and lighting. The introduction of fluid operating materials may cause drastic change in the intensity and illumination. Invariant structure information must be captured under different conditions. There are other moving objects in the scene that have significant interactions with the instruments and such as the new lens and the old lens fragments.

There has been limited previous work on localization and tracking of surgical instruments through image and video analysis, but in most of these cases the authors usually only deal with high quality images and very predicable patterns, or with virtual reality based surgery simulators[3], [4] and [5]. The developed system in this paper which is based on a robust multilevel tracking methodology, deals with the poor video quality and with the random characteristics of instruments. In addition it is fully real time as with $25 \mathrm{fps}$ as input it returns, after the analysis, an output frame rate of around $24.5 \mathrm{fps}$.

\section{DESCRIPTION OF THE INSTRUMENT TRACKING SYSTEM}

In the cataract surgical videos, the instrument tracking problems are complicated as discussed early on. Although there has been an attempt to make use of some of the commonly used object tracking techniques, none of these methods proved to be suitable for this application without major re-development. Many of published techniques may address one or two of listed issues but not all of them. On the other hand, this is a very interesting application environment that provides various challenges that can also benefit other similar applications and problems.

The proposed method is based on the very nature of the surgical data that the tracking algorithm has to deal with. Instead of trying to define an instrument and then try to track it, the main object that the system has to identify first is the eye. After the tracking algorithm locates the eye, it searches for instrument patterns inside.
The core algorithm of the instrument tracker is described in the InstrumentTracker procedure below:

\section{procedure InstrumentTracker}

1: If it is the first frame, prompt for the user to crop a part of the eye in order to build the model.

2: for each video frame do

3: Use the eye tracker module to locate the center of the eye.

4: Analyze the line patterns inside the eye mask, to find straight lines that could indicate the existence of an instrument.

5: Combine the information from the edge shape analysis with the change rate of the edges inside the eye to make the final decision if an instrument is present inside the eye or not.

6: If an instrument is present, record its position.

7: Update the eye model.

\section{8: end for}

The basic parts of the above algorithm are analyzed in more detail in subsections following.

\section{A. The eye tracking method}

The very key part in the instrument tracking system is the method to locate the position of the eye in order to define the region of interest (ROI) for the rest of analysis. To compromise the need for a real time system, the eye region and the background region are separated based on a threshold obtained from an initial pupil model. The model is constructed firstly through allowing the user to crop a small part of the pupil. The cropped sub-image is then converted from the RGB to HSV colour space. The values in S plane and $\mathrm{G}$ plane are analysed and corresponding thresholds are extracted for later locating ROI. The choice of the $\mathrm{S}$ plane is based on the fact that the pupil pixels have similar saturation values even if the colour changes rapidly during the surgery. The choice of the B plane, is for an extra level of check, as sometimes the $\mathrm{S}$ plane is heavily affected by the type of noise presented on these cataract videos.

One of the main hurdles for the SUITS system comes from the fact that the colour of the eye does not remain the same during the whole operation. This is due to changes in the illumination, changes in the angle of the camera, and finally due to some procedures of the surgeon, which alter the content of the eye (e.g. a cut with the scalpel that makes the eye red because of the blood in the vessels).

To overcome this problem, the model of the eye has to be constantly updated, so that it follows the new values and present better thresholding results. In order to constantly update the eye model, the information from the mean values of the $d$ previous frames have to be considered. The greater the value of $d$ is, the slower the system adapts to the eye changes. On the contrary, if the value of $d$ is very low, there is a risk that the model is changed so rapidly, that finally it loses the ability to track the eye.

Although the pupil blob is not the only one that remains after the thresholding process, it can easily be distinguished mathematically by extracting the compactness values of all 
the blobs. Assuming each blob $i$ has area of $A_{i}$ and perimeter $P_{i}$, the compactness is defined as:

$$
C_{i}=\frac{P_{i}^{2}}{A_{i}} \geq 4 \pi
$$

The value of $4 \pi$ is the lower value possible for the case of a perfect circle. So the blob that represents the eye is regarded to be the one that has the lower $C_{i}$ value.

A detailed block diagram explaining the basic operating principles of the SUITS is presented on Fig. 1. and an example of the output of the first steps on Fig. 2.

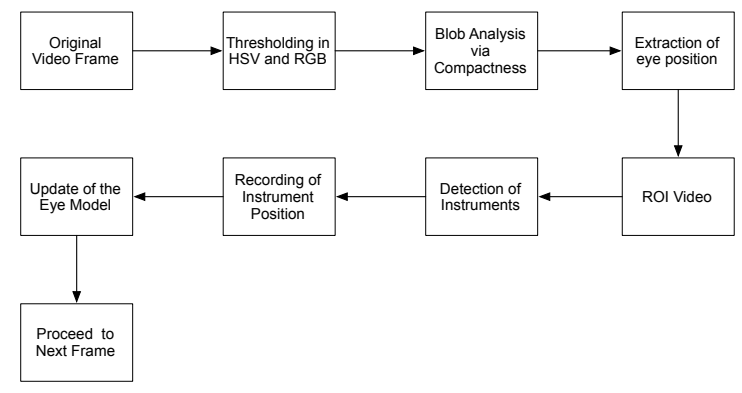

Fig. 1. Flow chart with the basic parts of the SUITS system.

After the eye tracking is completed, in order to proceed to the instrument tracking method, the edges of the ROI eye image have to be extracted. The results of the edge detection algorithms though were not as good as expected, due to the heavy presence of glare on almost every stage of the surgery videos. In order to make the instrument tracker more robust and minimize the false positives, a glare removal process is done that is described below.

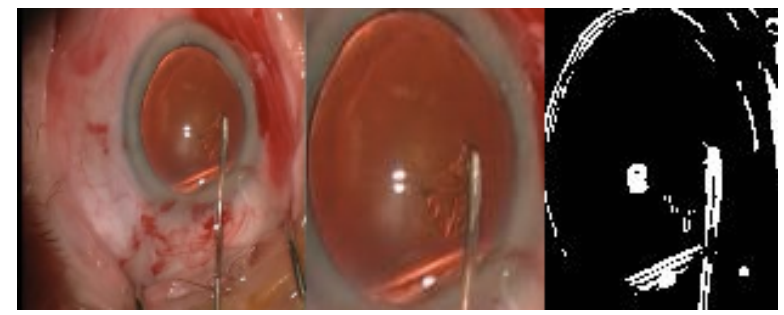

Fig. 2. From left to right we can see the original video frame, the result of the automatic iris ROI extraction, and the final edge image of interest.

The glare removal is taking place on the CIE-XYZ colour space [6]. Experimentally we discovered that this colour space is the most appropriate for this purpose. Specifically, the pixels of the the $\mathrm{Z}$ plane, can be easily classified to two categories glare pixels and non glare pixels. The non glare pixels values belong in the $[0,50]$ range, while the glare pixels on the $[200,255]$ range. By thresholding the intensity values of the $\mathrm{Z}$ plane we create a binary mask which corresponds to the glare areas of the video. This mask can be later used to exclude those glare pixels from the analysis.

\section{B. Locating an instrument inside the eye mask}

The method to decide if an instrument is present in the eye and then record its coordinates, is presented below. The straight line detection can be done either by using the well known hough algorithm or with the algorithm described on [7].

\section{procedureRecordInstrumentPosition}

1: if Straight line detection algorithm returns a line in the frame then

2: if Line ending or starting point is inside the eye mask then

3: $\quad$ Instrument is present in the eye.

Record it's X,Y coordinates.

\section{4: $\quad$ end if}

5: else

6: $\quad$ Proceed to the next frame.

No instrument was found.

7: end if

\section{Tool insertion and retraction decision method}

After the eye tracking algorithm returns the ROI of the eye, the next step is to search inside this region for straight lines, something that would indicate the existence of an instrument. To decide whether an instrument has entered the eye or not, two factors are taken into consideration. First of all, the presence of a straight line inside the ROI mask that could indicate an instrument. A second factor, is a gradual change in the EyeEdgesDensity $(\rho)$ quantity. This can be defined as the

$$
\rho=\frac{\text { Number of mask pixels belonging to an edge }}{\text { Number of total mask pixels }}
$$

Storing the $\rho$ values for every frame, a vector $\rho(i)$ can be constructed. After the construction of this vector, the change rate in the edges density can be expressed with the first derivative of this vector:

$$
\frac{d \rho(i)}{d i}
$$

$\frac{d \rho(i)}{d i}>0 \longrightarrow$ an instrument is entering the eye
$\frac{d \rho(i)}{d i}<0 \longrightarrow$ an instrument is retracted from the eye

\section{RESUlts AND EVAluation}

The main scope of SUITS is to provide information about the dexterity of the surgeon and the quality of the surgery. In [2], the variables that are taken into consideration are the total length of the instrument path, the total number of hand movements, and finally the total time. Another factor that can be used is the speed of the surgeon. However this metric alone may not always represent in the best possible way the dexterity, as the speed in order to be a positive feature has to be combined with precision.

\section{A. The total time that instruments are inside the eye region}

The first factor that is computed is the total time that the surgeon needs to complete the surgery. Since we know the video speed is $f$ fps (frames per second) we can find the total number of time in seconds that a surgeon has an instrument inside the eye. This is given by

$$
\text { Time }=\frac{F}{f} \text { seconds }
$$


where $F$ is the total number of frames with detected instrument inside.

\section{B. The surgery spectrogram}

Assuming that the $\rho$ values for every frame are computed, the vector $\rho(i)$ that was presented above can be used to produce a figure called surgery spectrogram (SS). We define the $\mathrm{SS}$ as the plot of the this $\rho(i)$ vector over time. A sample $\mathrm{SS}$ is presented on Figure 3.

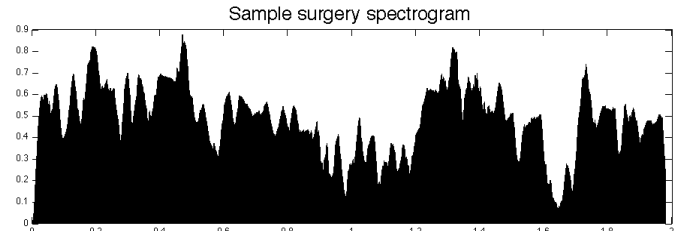

Fig. 3. A sample surgery spectrogram. The graph represents the edges density change inside the eye over time.

Analysis of this spectrogram, can provide valuable information about the surgery. Steady rise in this spectrogram over a period of time represents an instrument which is inserted in the eye, and steady fall implies that the instrument is retracted from the eye. Because of the heavy amount of noise that is present in the videos that were available for analysis, the result is sometimes difficult to distinguish, but for a noiseless video, the results are always expected to be good.

\section{Evaluation}

The results of the automated system that was developed were compared to a database of 20 videos, along with the number of surgeries the surgeon of each video has performed. However, not all videos were usable as the amount of noise present in many of them made the system fail to locate in detail and track the eye and the instruments. The doctors were divided in three categories, according to their experience which are Novice $(\approx 100$ surgeries $)$, intermediate $(\approx 500$ surgeries $)$, and experienced $(\approx 1000$ surgeries $)$. The 6 videos with the best quality ( 2 from each category) were chosen for analysis.

The metric that was used for the evaluation was a factor is called "surgery efficiency" $(\varepsilon)$ and is defined as the amount of pixels per move for every surgeon. The formula is

$$
\varepsilon=\frac{p a t h_{n}}{n}
$$

where $n$ is the number of movements taken into consideration, and path $_{n}$ is the total path of the surgeon for these $\mathrm{n}$ movements.

The defined quantity $\varepsilon$ in fact represents the "efficiency" of the surgeon. It is logical to assume that a novice doctor will present lower $\varepsilon$ value than an experienced one, for the same number of movements. This is because the experienced doctor is expected to achieve the same result (i.e. path) with less movements.

The results of the 6 videos from the database, in fact confirm the above hypothesis. In Figure 4, the plot of the $\varepsilon$ value for the 6 surgeons is presented. The graphs are sorted from left to right according to ascending experience. As we can see, there is a correlation between the experience of the surgeon, and his efficiency factor.

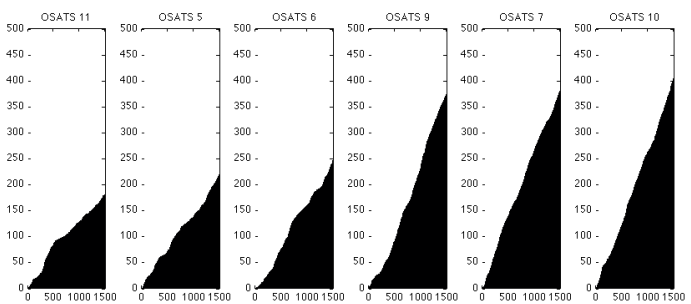

Fig. 4. "Efficiency factor" $(\varepsilon)$ for different levels of surgeon's experience. It is clear that the more experienced the surgeon is (in this graph the doctor's experience rises from left to right), the higher the value of $\varepsilon$.

\section{CONCLUSIONS AND FUTURE WORK}

The aim of the developed SUITS project is to produce a computational system that analyzes video data from cataract surgeries and provides feedback about surgeons experience. After the whole surgery video data are analyzed, the results are extracted for the computation of mathematical quantities related to the surgeons dexterity. The developed system is automatic, with minimal human supervision required throughout the processing time.

With the method described in this project, a new level of accuracy can be achieved which can be valuable for the medical community. Such a system would be useful both as an educational tool for novice surgeons and a valuable tool for the experienced ones. For example, inexperienced surgeons could compare their surgery statistics with others at the same level and learn their weakness. This may potentially revolutionise the surgical skill assessment. The developed system is a contribution to the further attempt to standardise the methods of medical training and analysis through objective criteria. There are some further issues and functions to be addressed and developed, such as the 3D measure of the movement and the ability to track multiple instruments within the eye.

\section{REFERENCES}

[1] Ellis H. Famous operations media. Pa: Harwal, 1984.

[2] George M. Saleh, Yiorgos Voyazis, Julian Hance, Joel Ratnasothy, and Ara Darzi. Evaluating surgical dexterity during corneal suturing. arch ophthalmol, 124(9):12631266, 2006.

[3] Bing Liu, Dennis Maier, Markus Schill, and Reinhard Maenner. Robust real-time localization of surgical instruments in the eye surgery simulator (eyesi).

[4] Sandrine Voros, Jean-Alexandre Long, and Philippe Cinquin. Automatic localization of laparoscopic instruments for the visual servoing of an endoscopic camera holder. In MICCAI (1), pages 535-542, 2006

[5] C. Doignon, P. Graebling, and M. de Mathelin. Real-time segmentation of surgical instruments inside the abdominal cavity using a joint hue saturation color feature. Real-Time Imaging, 11(5-6):429-442, 2005.

[6] B. Hill, Th. Roger, and F. W. Vorhagen. Comparative analysis of the quantization of color spaces on the basis of the cielab color-difference formula. ACM Trans. Graph., 16(2):109-154, 1997.

[7] Jinwen Ma and Lei Li. Automatic straight line detection through fixed-point byy harmony learning. In ICIC '08: Proceedings of the 4th international conference on Intelligent Computing, pages 569-576, Berlin, Heidelberg, 2008. Springer-Verlag. 